\title{
Antitumor Effects of a Human Dimeric Antibody Fragment 131I-AFRA-DFM5.3 in a Mouse Model for Ovarian Cancer
}

\author{
Alberto Zacchetti ${ }^{1}$, Franck Martin ${ }^{2}$, Elena Luison ${ }^{1}$, Angela Coliva $^{3}$, Emilio Bombardieri ${ }^{3}$, Marcello Allegretti ${ }^{2}$, \\ Mariangela Figini*1, and Silvana Canevari*1 \\ ${ }^{1}$ Unit of Molecular Therapies, Department of Experimental Oncology and Molecular Medicine, Fondazione IRCCS Istituto Nazionale \\ dei Tumori, Milan, Italy; ${ }^{2}$ Dompé S.P.A., L'Aquila, Italy; and ${ }^{3}$ Department of Imaging and Nuclear Medicine, Fondazione IRCCS \\ Istituto Nazionale dei Tumori, Milan, Italy
}

\begin{abstract}
AFRA-DMF5.3 is a human antibody fragment that, as a dimer, specifically binds to the $\alpha$-folate receptor (FR) on ovary cancer cells. Pharmacokinetic and biodistribution parameters of ${ }^{131}$ I-AFRADFM5.3 after intravenous administration in animal models support its potential therapeutic use. We evaluated its preclinical specificity and therapeutic efficacy in tumor models. Methods: A negative control, AFRA-DFM6.1, was obtained by protein engineering. The activity and specificity of ${ }^{131}$ I-AFRA-DFMs were evaluated by systemic administration (intravenous) in subcutaneous tumor xenograft-bearing nude mice. Pharmacokinetics, biodistribution, and efficacy were assessed by intraperitoneal administration of ${ }^{131}$ I-AFRA-DFM5.3 in nude mice bearing 2 different intraperitoneal ovarian carcinoma xenografts. Treatments were tested at different doses and as single or double administrations $1 \mathrm{wk}$ apart. Results: In subcutaneous models, ${ }^{131}$ I-AFRA-DFM5.3, but not the negative control, was found to reside on FR-positive tumor masses and significantly reduced tumor growth. In intraperitoneal models, early accumulation on free-floating clumps of ovarian cancer cells and solid peritoneal masses was evident after $1 \mathrm{~h}$, and tumor uptake was stable for up to $3 \mathrm{~h}$. The high tumor uptake determined the efficacy of ${ }^{131} \mathrm{I}$-AFRA-DFM5.3. The best antitumor activity, with more than $50 \%$ of treated animals cured, was achieved with 2 locoregional treatments of intraperitoneally growing tumors on days 2 and 9. Conclusion: These results suggest that radioimmunotherapy with ${ }^{131}$ I-AFRA-DFM5.3 is feasible and leads to significantly prolonged survival. These preclinical data provide the basis for the rationale design of therapeutic treatments of ovarian cancer patients with a radiolabeled anti-FR antibody fragment.
\end{abstract}

Key Words: ovarian cancer; folate receptor; human antibody fragment; experimental radioimmunotherapy

J Nucl Med 2011; 52:1938-1946

DOI: 10.2967/jnumed.110.086819

Received Dec. 17, 2010; revision accepted Jul. 5, 2011.

For correspondence or reprints contact: Mariangela Figini, Department of Experimental Oncology, Fondazione IRCCS Istituto Nazionale dei Tumori, Via Venezian 1, 20133 Milan, Italy.

E-mail: mariangela.figini@istitutotumori.mi.it

${ }^{*}$ Contributed equally to this work.

Published online Nov. 8, 2011.

COPYRIGHT (c) 2011 by the Society of Nuclear Medicine, Inc.
$\mathbf{O}$ varian cancer is the leading cause of death for patients with gynecologic malignancies (1). A major problem in clinical management of ovarian cancer is the unpredictable response to first-line treatment and the high frequency of relapse after induction chemotherapy, associated with broad cross-resistance to structurally dissimilar drugs. This problem highlights the need for anticancer treatments with mechanisms of cancer toxicity that are different from those of currently available chemotherapeutic agents.

Selective exploitation of molecules that are aberrant in cancer using targeted anticancer treatment appears successful in a variety of malignancies, such as breast, colon, lung, and renal cancers. Numerous targeted therapeutic approaches have also been explored in ovarian cancer using either small inhibitors of tyrosine kinases or monoclonal antibodies (mAbs) (2). Numerous ongoing randomized trials have investigated a mAb as the targeted agent, but at present no consensus therapy with this class of molecules has been reached for ovarian cancer.

Antibody-based therapies are promising (3), and as recently discussed (4), an interesting application is radioimmunotherapy. In fact, radioimmunotherapy may be superior to antibody alone or to antibody conjugated with drugs or toxins because of the cross-fire phenomenon $(5,6)$ and the relative independence from the homogeneous expression of the target within the tumor mass.

In recent years, radioimmunotherapy has become a consolidated therapeutic option for hematologic malignancies, whereas its application to solid tumors has been progressing more slowly (7). The limited effects of radioimmunotherapy in solid tumors, and, in general, of therapies based on entire antibody molecules, can be attributed to a low tumor-mass penetration, which depends on the dimension and vascularization of the tumor as well as intrinsic chemical-physical features of the antibody, such as size, charge, and species of origin (8). Antibody fragments obtained by protein engineering and the use of locoregional treatments for some malignancies, such as intrathecal injection in glioblastoma patients and intra- 
peritoneal delivery in ovarian cancer patients, hold promise for overcoming some of the limitations due to poor tumor penetration.

In the case of ovarian cancer, even though heavily debated, locoregional administration of treatment has a strong biologic and pharmacologic rationale. In fact, intraperitoneal spread is the most characteristic feature of ovarian cancer metastases, and residual tumor after standard aggressive surgery is primarily confined to the peritoneal cavity. Pharmacodynamic studies have shown that intraperitoneal chemotherapy can achieve higher local concentrations and prolonged drug exposure $(9,10)$.

In the 1990s, encouraging results were obtained in phase I and II trials with anti-folate receptor (FR) murine antibody derivatives administered intraperitoneally in ovarian cancer patients (11-13). The major limitation of these studies was that the resulting human antimouse antibody response did not allow for repeated injection. Genetic engineering, however, allowed the generation of chimeric (chi) antibodies (murine-variable regions and human constant regions). Chi-MOv18 (14) entered in preclinical and clinical studies with particular attention to radioimmunotherapy applications $(15,16)$, including intraperitoneal administration $(17,18)$. In the case of chi antibodies, patients eventually tend to develop levels of human antimouse antibody that are comparable to those observed with mouse antibodies. Human antibodies could be an ideal reagent for this purpose. In the past, we have produced different human antibodies against FR using phage display, starting either from a naïve library (19) or from a library from patients with a previous history of ovarian cancer (4) using guided selection, which allows for isolation of an antibody with the same specificity as the preexisting murine antibody. In particular, several antibody fragments have been isolated, one of which was genetically and chemically manipulated to obtain a dimer, namely AFRA-DFM5.3, with characteristics suitable for clinical applications $(4,20)$.

Another limitation of intraperitoneal immunotherapy against FR was the relatively long half-life of ${ }^{131} \mathrm{I}$-antiFR murine (11) or chi (18) mAbs. In contrast, the pharmacokinetic parameters of the human antibody fragment ${ }^{131}$ I-AFRA-DFM5.3 observed in well-defined preclinical animal models (4) support its potential therapeutic use, not only as a single injection but also when administered 2 times 1 wk apart.

\section{MATERIALS AND METHODS}

\section{Cell Lines and Antibodies}

The following human tumor cell lines were used: ovarian carcinoma IGROV-1 (a gift from Dr. Jean Bénard, Institute Gustave Roussy), OVCAR3 (American Type Culture Collection), and A431FR and A431-MK (epidermoid carcinoma cells transfected with FR or empty vector, respectively), isolated as previously described (21). All cell lines were grown in RPMI 1640 medium supplemented with $10 \%$ fetal calf serum and $2 \mathrm{mM}$ glutamine; stably transfected cells were grown with the addition of G418 at $800 \mu \mathrm{g} / \mathrm{mL}$ (Gibco).
The level of FR expression was assessed by Western blot analysis and defined using an arbitrary scale: moderate for IGROV-1, high for OVCAR3, and very high for A431FR (4).

For in vivo studies, the following antibodies were used: AFRADFM5.3 (4), human anti-FR antibody Fab dimer (final concentration, $0.13 \mathrm{mM}$ ); AFRA-DFM6.1, human nonbinder antibody Fab dimer (final concentration, $0.05 \mathrm{mM}$ ); and chi-MOv18 $\mathrm{Fab}_{2}{ }_{2}$, prepared using the ImmunoPure $\mathrm{Fab}_{2}{ }_{2}$ Preparation Kit [Pierce]) (final concentration, $0.07 \mathrm{mM}$ ), chosen on the basis of similarity in format and already used under a preclinical analysis (15). Antibodies were produced in clinical grade conditions and verified for sterility and pyrogens below the level of detection (4). The binding affinity of AFRA-DFM5.3, AFRA-DFM6.1, and chi-MOv18 $\mathrm{Fab}_{2}{ }_{2}$ was determined using the Biacore system as previously described (4).

A reference entire human $\mathrm{mAb}$ and its $\mathrm{F}(\mathrm{ab})_{2}$ fragment and an anti-human fluorescein-labeled secondary antibody (goat; KPL) were used for isoelectrofocusing and fluorescence-activated cell sorting, respectively.

\section{Generation of Nonbinder Reagent}

To disrupt the binding ability of the AFRA5.3 Fab fragment, its heavy chain was modified by site-directed mutagenesis of DNA triplet codons to obtain a fragment termed AFRA6.1.

\section{Radiolabeling and Quality Control}

${ }^{131}$ I was from GE Healthcare; all other chemicals were of high purity (Sigma). Radiolabeling with ${ }^{131}$ I was done using IODOGEN-precoated iodination tubes (Pierce) by the Chizzonite method, as previously described $(4,21)$. Radiopharmaceuticals were characterized for radiochemical purity by instant thin layer chromatography, integrity by sodium dodecyl sulfate polyacrylamide gel electrophoresis, isoelectric point (pI) by isoelectrofocusing using the Phast System (GE Healthcare), and immunoreactivity as previously described (4), using A431FR, IGROV-1, or OVCAR3 as positive target cells and A431MK as negative target cells.

\section{Animals and Tumor Models}

All protocols were approved by the Ethics Committee for Animal Experimentation of the Fondazione IRCCS Istituto Nazionale dei Tumori and performed according to institutional and the new Guidelines for the Welfare and Use of Animals in Cancer Research (22). Female s.c.e CD1 nu/nu (athymic) mice were obtained at 6-7 wk of age from Charles River Laboratories and left untreated for at least 1 wk of acclimatization. Subcutaneous models were chosen to define the specificity of the radioimmunotherapy with easy quantification of tumor dimension. Toward this end, and because of the poor subcutaneous tumor uptake of the ovarian cancer cell lines implanted, 2 isogenic cell lines differing only in the presence or absence of the antigen of interest (A431FR and A431MK) were used; mice were injected subcutaneously in the flank region with $3.5 \times 10^{6}$ tumor cells in $0.1 \mathrm{~mL}$ of saline. For evaluation of efficacy, intraperitoneal models of ovarian cancer cells, namely IGROV-1 and OVCAR3 cells adapted to grow intraperitoneally and maintained by serial intraperitoneal passages of ascitic cells $(23,24)$, were used; mice were injected intraperitoneally with $1 \times 10^{7}$ tumor cells in $0.5 \mathrm{~mL}$ of saline. Hemorrhagic ascites with diffuse peritoneal carcinomatosis developed in about $15 \mathrm{~d}$ in all mice of the OVCAR3 model and around $50 \%$ of the mice of the IGROV-1 model; in all ascites-bearing animals, solid tumor masses were also detected at necropsy in the peritoneal cavity and in IGROV-1-bearing mice without ascites. A major intraperitoneal nodule located on 
the intestinal mesentery or on the peritoneal wall was recorded by palpation or at necropsy. The following were humane endpoints: for subcutaneous models, tumor volume greater than $750 \mathrm{~mm}^{3}$ or body weight loss greater than $20 \%$, and for intraperitoneal models, body weight increase greater than $20 \%$ or intraperitoneal tumor mass with an estimated volume greater than $500 \mathrm{~mm}^{3}$.

\section{In Vivo Toxicity}

The maximal AFRA-DFM-injected doses were selected according to previous experience with ${ }^{131}$ I-labeled antibodies by us (20) and others (23) and taking into consideration both operator exposure and possible initial cross-irradiation between animals. Possible animal death due to irradiation (monitored in non-tumor-bearing mice) and body weight were controlled on a regular basis.

\section{Systemic Treatment}

At day 6-7 after subcutaneous tumor injection, mice with growing tumors (mean tumor volume \pm SD: A431FR, $32 \pm$ $18 \mathrm{~mm}^{3}$; A431MK, $35 \pm 15 \mathrm{~mm}^{3}$ ) were randomly divided into groups $(n=6-8)$ and injected in the lateral tail vein with $0.3 \mathrm{~mL}$

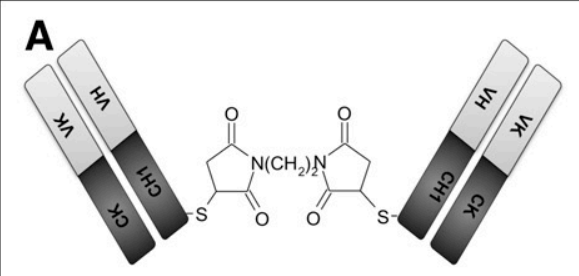

AFRA-DFM 5.3

B

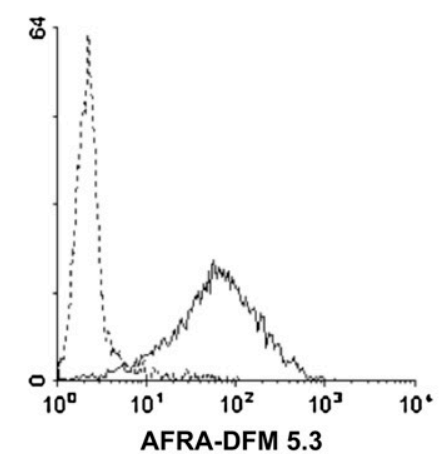

C

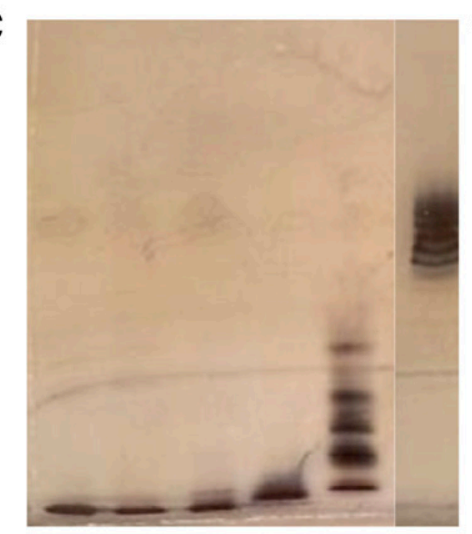

$-\mathrm{pH} 9$

$-\mathrm{pH} 3$

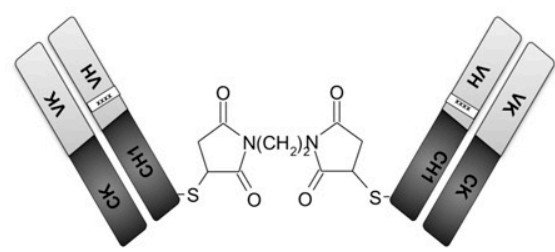

AFRA-DFM 6.1

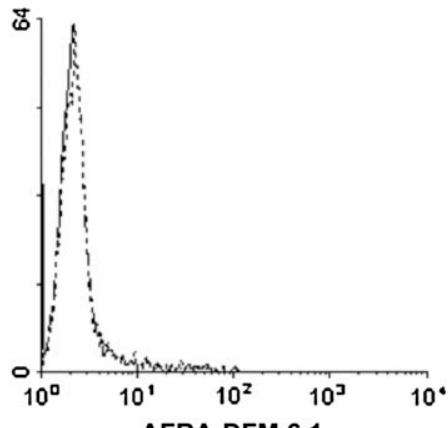

AFRA-DFM 6.1

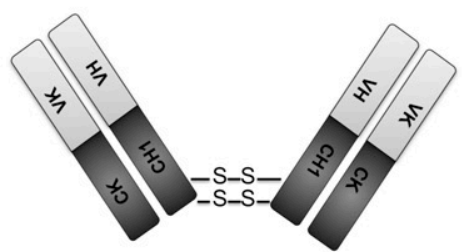

Chi Mov18 F(ab')2
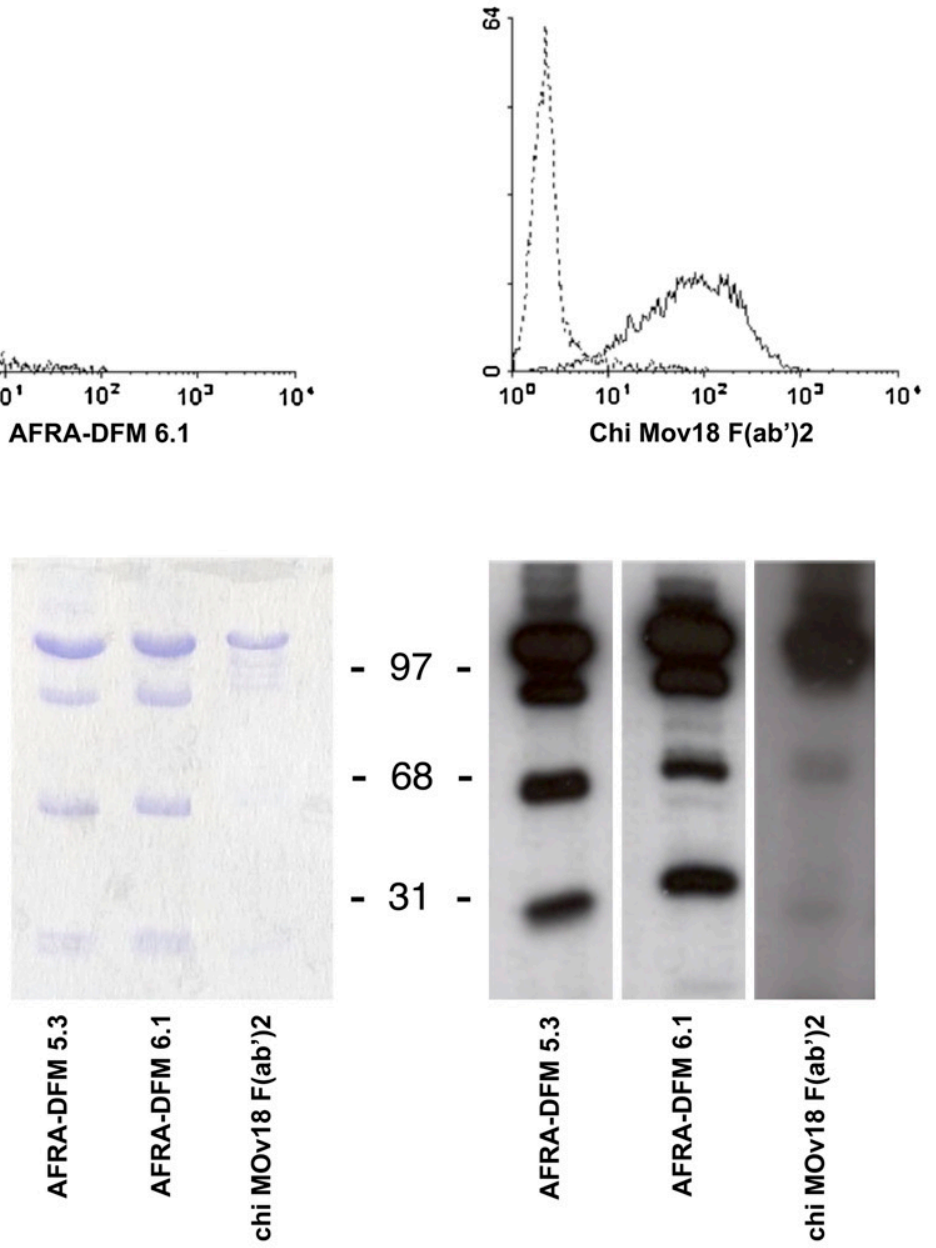

FIGURE 1. Immunochemical characterization of AFRA-DFM5.3, compared with controls. (A) Schematic diagram of antibody fragments used. (B) Binding profiles of AFRA-DFM5.3, AFRA-DFM6.1, and chi-MOv18 (Fab) $)_{2}$ to A431FR assessed by fluorescence-activated cell sorting. (C) Isoelectric point determination of AFRA-DFM5.3, AFRA-DFM6.1, and chi-MOv18 (Fab) $)_{2}$ by isoelectrofocusing (3-9 pl) and silver staining; reference human entire $\mathrm{mAb}$ and its $\mathrm{F}(\mathrm{ab})_{2}$ fragment and murine entire mAb MOv18 were used. (D) Analysis of purified AFRA-DFMs and chi-MOv18 (Fab) ${ }_{2}$ before (left) and after (right) ${ }^{131} \mathrm{I}$-radiolabeling by sodium dodecyl sulfate polyacrylamide gel electrophoresis (12.5\%) in nonreducing conditions and detected by either staining with Coomassie blue or autoradiography. $\mathrm{h}=$ human; Ref. $=$ reference. 
of ${ }^{131}$ I-AFRA-DFMs or ${ }^{131}$ I-chi-MOv18 $(\mathrm{Fab})_{2}$ in saline or saline alone as a control. Tumor growth was monitored with a caliper every 2-3 d, and volume was calculated as $V=4 / 3 \pi r^{3}$. Tumor growth is reported as a logarithm of the mean relative tumor volume $\left(\log _{10}\right.$ of the ratio of tumor volume at each time point to tumor volume at the day of radioconjugate injection) \pm SD (22).

\section{Intraperitoneal Biodistribution}

At day 10-14 after intraperitoneal tumor injection, groups of 3-4 animals for each time point were intraperitoneally injected with $0.3 \mathrm{~mL}$ of ${ }^{131} \mathrm{I}$-AFRA-DFMs $(1.1 \mathrm{MBq})$. For pharmacokinetic and biodistribution studies, at 10 and $30 \mathrm{~min}$ and at 1, 3, 6, and $15 \mathrm{~h}$, blood samples, selected tissues or organs, ascitic fluid, and intraperitoneal tumor masses were harvested, weighed, and counted in a $\gamma$-counter to calculate the percentage of the injected dose per gram of tissue $(\% \mathrm{ID} / \mathrm{g})$.

\section{Locoregional Treatment}

At day 2 or 4 after intraperitoneal tumor injection, animals were randomly divided into groups $(n=4-8)$ and intraperitoneally injected with $0.3 \mathrm{~mL}$ of ${ }^{131}$ I-AFRA-DFM5.3 in saline or with saline alone as a control. Animals were monitored for body weight, ascites development, and intraperitoneal tumor masses every $2-3$ d. At the end of the experiments, surviving animals were euthanized, and necropsy was performed to evaluate tumor dissemination in the peritoneal cavity.

\section{Statistical Analysis}

Prism (version 5.02; GraphPad Software) was used for statistical analysis. In subcutaneous tumor models, the activity and specificity of systemic treatment were assessed by comparing the mean tumor volume and weight at the end of the experiment with an unpaired 2-tailed Student $t$ test. In the intraperitoneal tumor models, the efficacy of the locoregional treatment was assessed by comparing the overall survival by log-rank assay using the Kaplan-Meier product-limit method. The Cox univariate model was used to estimate the hazard ratio. A $P$ value of less than 0.05 was considered statistically significant.

\section{RESULTS}

\section{In Vitro Selection and Characterization of Negative and Positive Antibody Controls}

To obtain an appropriate negative control, we engineered an antibody-based reagent with the same chemical-physical characteristics as AFRA5.3 but unable to bind FR. Four changes in the amino acid sequence of CDR3 of the heavy variable chain of AFRA5.3 and 2 changes, for cloning reasons, in the framework 4 region were sufficient to obtain AFRA6.1. By fluorescence-activated cell sorting analysis (Fig. 1A), AFRA-DFM5.3 and chi-MOv18 Fab' $_{2}$ exhibited a binding profile consistent with their specific recognition of FR, whereas no binding activity to FR-expressing A431FR was detectable for AFRA-DFM6.1. Neither antibody reagent bound to A431MK cells not expressing FR (data not shown). BIAcore analysis confirmed that AFRA-DFM6.1 does not bind to recombinant purified FR (data not shown).

Both the human AFRA-DFMs, similar to the human reference entire antibody and human fragment, had a pI higher than 9, whereas the chi-MOv18-F(ab) 2 and the murine antibody MOv18 showed a pI of around 8.5 and 6.8, respectively (Fig. 1B).

AFRA-DFMs and chi-MOv18 $(\mathrm{Fab})_{2}$ were radiolabeled with ${ }^{131}$ I using similar experimental conditions without alteration of molecular integrity (Fig. 1C). Analysis of immunoreactivity indicated that greater than $70 \%$ and $55 \%$ of the radiolabeled anti-FR reagents AFRA-DFM5.3 and chiMOv18 (Fab) ${ }_{2}$ bound to cells overexpressing FR, whereas no binding over background was observed with radiolabeled AFRA-DFM6.1 (mean immunoreactivity $<1 \%$ ).

\section{In Vivo Characterization of ${ }^{131}$ |-AFRA-DFMs}

The blood terminal half-life of ${ }^{131}$ I-AFRA-DFM6.1 after intravenous administration in non-tumor-bearing mice and

TABLE 1

Antitumor Activity of ${ }^{131}$ I-Labeled Anti-FR Antibodies After Systemic Administration in Subcutaneous Tumor Models

\begin{tabular}{|c|c|c|c|c|c|c|c|c|c|}
\hline \multirow[b]{2}{*}{$\begin{array}{c}\text { Experiment } \\
\text { no. }\end{array}$} & \multirow[b]{2}{*}{$\begin{array}{l}\text { Radiolabelled } \\
\text { reagent }\end{array}$} & \multirow[b]{2}{*}{$\begin{array}{l}\text { Total dose } \\
(\mathrm{MBq})\end{array}$} & \multirow[b]{2}{*}{$\begin{array}{l}\text { Days of } \\
\text { treatment }\end{array}$} & \multirow[b]{2}{*}{ Tumor } & \multirow[b]{2}{*}{$\begin{array}{l}\text { Antigen } \\
\text { expression }\end{array}$} & \multicolumn{4}{|c|}{ Antitumor activity over untreated animals } \\
\hline & & & & & & $\begin{array}{c}\text { Percentage tumor } \\
\text { volume }^{\star}\end{array}$ & $P$ & $\begin{array}{l}\text { Percentage } \\
\text { tumor weight* }\end{array}$ & $P$ \\
\hline \multirow[t]{2}{*}{1} & $\begin{array}{l}\text { AFRA-DFM5.3 } \\
\text { (Fig. 2A) }\end{array}$ & 37 & +6 & A431FR & Positive & 38 & $<0.0001$ & 49 & 0.0303 \\
\hline & & 37 & +6 & A431MK & Negative & 93 & 0.3461 & 82 & 0.1354 \\
\hline 2 & AFRA-DFM6.1 & 37 & +6 & A431FR & Positive & 90 & 0.6422 & 81 & 0.3455 \\
\hline \multirow[t]{2}{*}{3} & $\begin{array}{l}\text { AFRA-DFM5.3 } \\
\text { (Fig. 2B) }\end{array}$ & 74 & +7 and +13 & A431FR & Positive & 37 & $<0.0001$ & 44 & 0.0015 \\
\hline & & 74 & +7 and +13 & A431MK & Negative & 76 & 0.2391 & 70 & 0.0637 \\
\hline 4 & AFRA-DFM6.1 & 74 & +7 and +13 & A431FR & Positive & 81 & 0.3186 & 72 & 0.1381 \\
\hline \multirow[t]{2}{*}{5} & $\begin{array}{l}\text { chi-MOv18 Fab2 } \\
\text { (Fig. 2C) }\end{array}$ & 37 & +7 & A431FR & Positive & 11 & 0.0005 & 18 & 0.0002 \\
\hline & & 37 & +7 & A431MK & Negative & 81 & 0.5090 & 81 & 0.2960 \\
\hline
\end{tabular}

${ }^{*}$ Ratio of tumor volume or weight in treated vs. control mice $\times 100$ measured at experiment end; analyzed with unpaired Student $t$ test. Number of animals: treated, 6-7; controls, 6-8. Some intra- and interassay variability was observed in tumor volume and weight in control mice at 20-23 d after tumor injection: A431FR, 280-630 $\mathrm{mm}^{3}$ and 0.5-1.5 g; A431MK: 310-700 $\mathrm{mm}^{3}$ and $0.3-1.5 \mathrm{~g}$. 


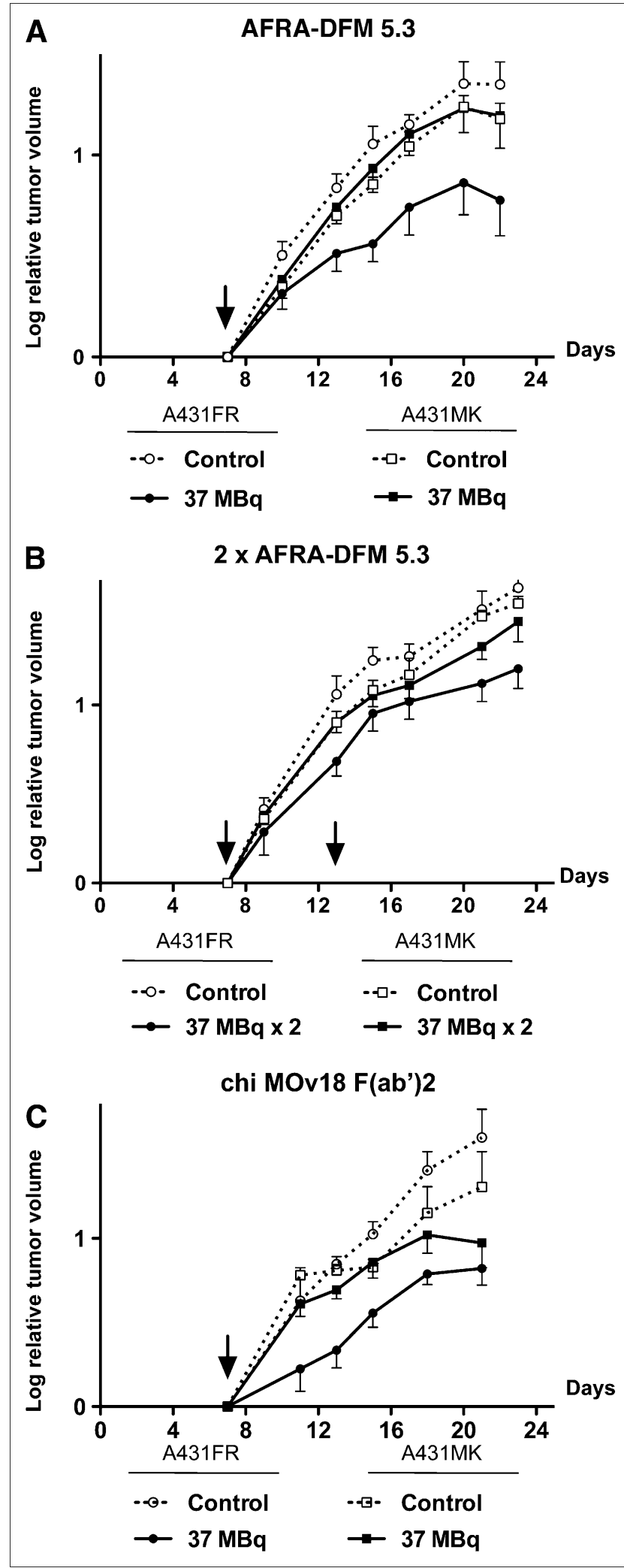

FIGURE 2. Efficacy and specificity of ${ }^{131}$ I-AFRA-DFM radioimmunotherapy administered by systemic injection in animals bearing subcutaneous A431FR or A431MK. (A) Single treatment with $37 \mathrm{MBq}$ of ${ }^{131} \mathrm{I}$-AFRA-DFM5.3. (B) Double treatment, $1 \mathrm{wk}$ apart, with $37 \mathrm{MBq}$ of ${ }^{131}$ I-AFRA-DFM5.3. (C) Single treatment with 37 $\mathrm{MBq}$ of ${ }^{131} \mathrm{I}$-chi-MOv18 $(\mathrm{Fab})_{2}$. Table 1 provides details and stat- in A431FR and A431MK tumor-bearing mice was similar to that previously reported for ${ }^{131}$ I-AFRA-DFM5.3 (4) and ranged between 3.3 and $3.7 \mathrm{~h}$. Similar results were obtained by intraperitoneal administration; in fact, the blood terminal half-life of the two ${ }^{131}$ I-AFRA-DFMs was comparable (3.2 vs. $3.3 \mathrm{~h}$ ) in mice not bearing tumors. Blood terminal halflife of ${ }^{131} \mathrm{I}$-chi-MOv18 (Fab) ${ }_{2}$ after intravenous administration in tumor-bearing mice ranged between 8.6 and $9.1 \mathrm{~h}$.

No major toxic effects of radiation were recorded in treated animals after systemic or locoregional administration of either $55 \mathrm{MBq}$ as a single injection or $74 \mathrm{MBq}$ injected in 2 doses $1 \mathrm{wk}$ apart.

\section{Systemic Treatment}

For evaluation of radioimmunotherapy activity and specificity, the 2 AFRA-DFM human fragments were tested after systemic administration in the subcutaneous model system previously used to evaluate the efficacy of the antiFR ${ }^{131}$ I-MOv18 antibody (20). Details of the experiments are reported in Table 1, and representative curves are shown in Figure 2.

When ${ }^{131}$ I-AFRA-DFM5.3 was administered once (Fig. 2A) in A431FR-bearing animals, a significant delay in tumor growth was observed, and final tumor volume and weight were significantly lower than in control animals; however, 2 doses (Fig. 2B) did not suggest that the therapeutic effect was better than that achieved by a single injection (final tumor volume and weights: $37 \%$ vs. $38 \%$ and $44 \%$ vs. $49 \%$ of controls, respectively). Moreover, no decrease or only a minor decrease in tumor growth and weight were recorded in FR-nonexpressing A431MK-bearing animals after a single (Fig. 2A) or repeated administration (Fig. 2B).

Radioimmunotherapy with the nonbinding antibody fragment ${ }^{131}$ I-AFRA-DFM6.1 administered once or twice exerted only a marginal delay in tumor growth (final tumor volume and weight that was 71\%-90\% of control values).

${ }^{131}$ I-chi-MOv18 (Fab) 2 (Fig. 2C) radioimmunotherapy resulted in a reduction of final tumor volume and weight of A431FR and A431MK tumor xenografts that, probably as a consequence of its slower clearance, was higher than that achievable with ${ }^{131}$ I-AFRA-DFM5.3.

\section{Intraperitoneal Biodistribution and Tumor Localization}

When ascites or intraperitoneal nodules were evident, the ${ }^{131}$ I-AFRA-DFM fragments were administered intraperitoneally. The blood terminal half-life of ${ }^{131}$ I-AFRADFM5.3 was longer than that of ${ }^{131}$ I-AFRA-DFM6.1 (4.4 \pm 0.6 vs. $3.3 \pm 0.2 \mathrm{~h}$, respectively). Accumulation of ${ }^{131} \mathrm{I}-$ AFRA-DFM5.3 on tumor cells present in ascites was rapid and maximal at $1 \mathrm{~h}$ after intraperitoneal injection, whereas the maximal accumulation on solid intraperitoneal tumor

istical analysis. Data are reported as $\log _{10}$ of mean relative tumor volume $\pm \mathrm{SD}$; arrows indicate day of treatment. 
masses and other tissues was observed after 3 and $6 \mathrm{~h}$ (Fig. 3A). In Figure 3B, the results obtained $3 \mathrm{~h}$ after intraperitoneal injection of ${ }^{131}$ I-AFRA-DFM fragments in the IGROV-1 intraperitoneal tumor model are shown, permitting precise evaluation of specificity in animals with or without ascitic fluid formation. Differences in solid tumor accumulation were observed between mice that developed only solid tumor masses or also ascites; however, although the difference was highly significant for AFRA-DFM5.3 $(P<0.001)$, only a trend toward higher accumulation was noted for AFRADFM6.1 $(P=0.0881)$. Furthermore, in animals with ascites, free-floating tumor cells presented a high uptake of the specific AFRA-DFM5.3 binder, compared with negligible accumulation of the nonbinder AFRA-DFM6.1 (mean \%ID/g \pm $\mathrm{SD}, 25.11 \pm 6.94$ and $0.23 \pm 0.05$, respectively). Despite the longer terminal half-life of ${ }^{131}$ I-AFRA-DFM5.3, the $\% \mathrm{ID} / \mathrm{g}$ in normal tissue or organs (data shown only for blood and kidneys) was similar to that seen for ${ }^{131}$ I-AFRA-DFM6.1.

\section{Locoregional Treatment}

The efficacy of locoregional radioimmunotherapy in controlling intraperitoneal IGROV-1 or OVCAR3 growth was analyzed by administration of ${ }^{131}$ I-AFRA-DFM5.3 2 or $4 \mathrm{~d}$ after tumor injections, when cells were already implanted in the peritoneal cavity but ascitic fluid formation was not yet detectable (Table 2). Representative survival curves are shown in Figure 4. In all experiments, the differences in survival of treated animals, compared with controls, were statistically significant.

Similar results were obtained after a single treatment with ${ }^{131}$ I-AFRA-DFM5.3 independent of the dose used (37 or $55 \mathrm{MBq}$ ). At necropsy, untreated animals presented both ascites and solid tumor masses, whereas the ${ }^{131}$ I-AFRADFM5.3-treated animals had only solid masses. A double treatment, 1 wk apart (Fig. 4 and Table 2), was able to cure around $50 \%$ of animals and prevent tumor development until the end of monitoring ( $3 \mathrm{mo}$ ). At necropsy, all animals that survived were macroscopically free of tumor.

\section{DISCUSSION}

In this preclinical study, we tested the hypothesis that an antibody fragment in a dimer format might be the reagent of choice for intraperitoneal radioimmunotherapy of ovarian cancer because of its relatively small size, which should favor tumor penetration and fast clearance. Furthermore, a

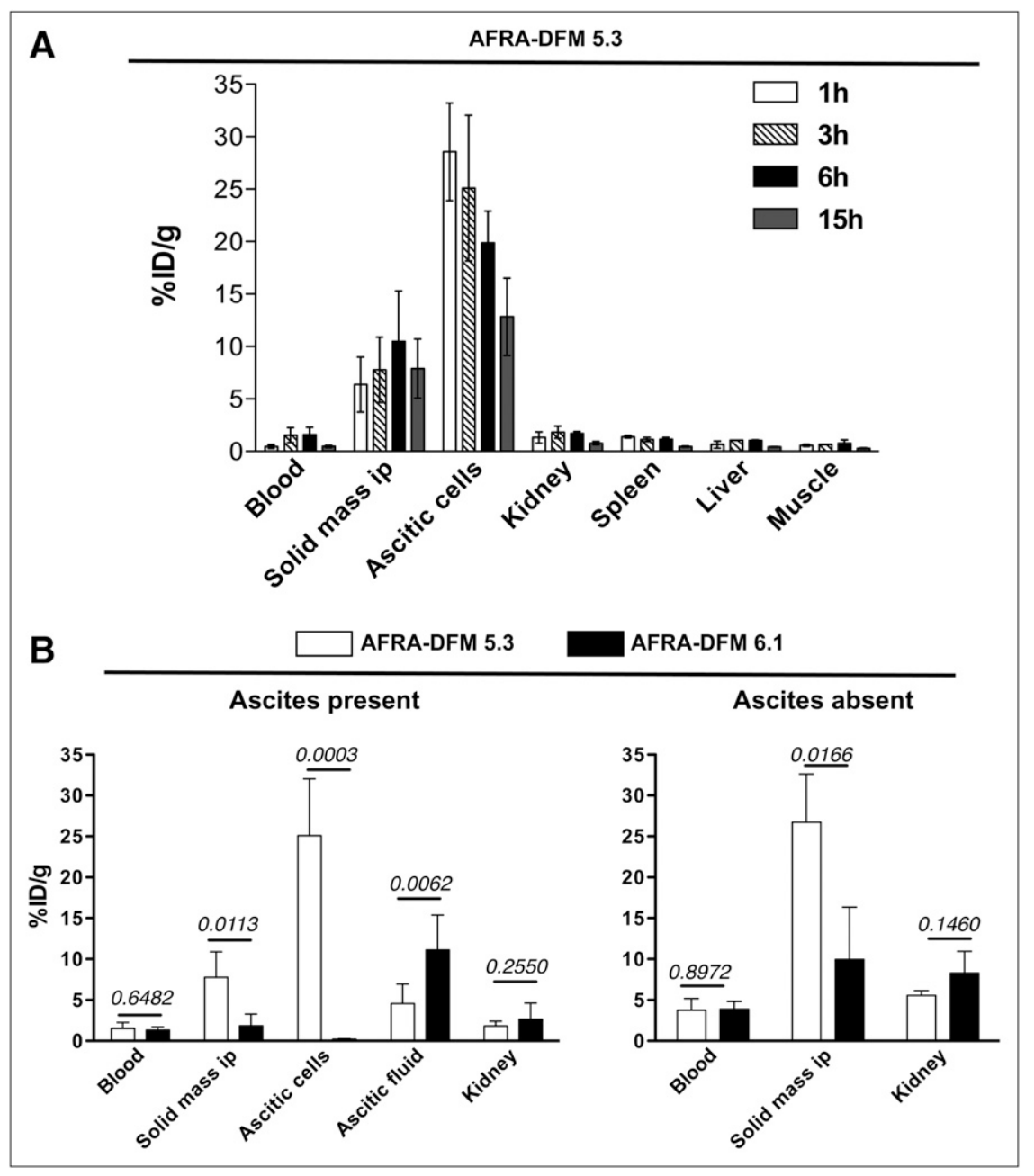

FIGURE 3. Biodistribution and tumor localization after locoregional injection of ${ }^{131}$ I-AFRA-DFMs in intraperitoneal IGROV1-bearing animals. (A) Time course of localization of ${ }^{131}$ I-AFRA-DFM5.3 as mean \%ID/ $\mathrm{g} \pm \mathrm{SD}$ in blood, ascitic cells, solid tumor mass, and representative normal organs after intraperitoneal administration; animals per time point $=3-4$. Representative experiment $(n=3)$ is shown. (B) ${ }^{131}$ I-AFRA-DFM5.3 and ${ }^{131}$ I-AFRA-DFM6.1 localization in selected tissue or organs of intraperitoneal IGROV-1-bearing animals $3 \mathrm{~h}$ after injection (mean of 2 experiments). Tumor ascitesbearing animals (mean volume $\pm \mathrm{SD}$ : total ascites, $5.3 \pm 2.5 \mathrm{~mL}$; cell pellet, $0.7 \pm 0.4$ $\mathrm{mL})$ were injected with ${ }^{131} \mathrm{I}$-AFRA-DFM5.3 $(n=6)$ or ${ }^{131}$ I-AFRA-DFM6.1 $(n=3)$. Tumorbearing animals without ascites were injected with ${ }^{131}$ I-AFRA-DFM5.3 $(n=3)$ or 131I-AFRA-DFM6.1 $(n=3)$. Major intraperitoneal solid mass was weighed (mean \pm $\mathrm{SD}, 186 \pm 82 \mathrm{mg}$ ). ip = intraperitoneal. 
TABLE 2

Antitumor Activity of Anti-FR ${ }^{131}$ I-Labeled AFRA-DFM5.3 After Locoregional Administration in Intraperitoneal Tumor Models

\begin{tabular}{|c|c|c|c|c|c|c|c|c|}
\hline \multirow[b]{2}{*}{$\begin{array}{c}\text { Experiment } \\
\text { no. }\end{array}$} & \multirow[b]{2}{*}{$\begin{array}{l}\text { Total } \\
\text { dose } \\
(\mathrm{MBq})\end{array}$} & \multirow[b]{2}{*}{$\begin{array}{l}\text { Days of } \\
\text { treatment }\end{array}$} & \multirow[b]{2}{*}{$\begin{array}{l}\text { Treated } \\
\text { Tumor }\end{array}$} & \multirow[b]{2}{*}{$\begin{array}{l}\text { No. of } \\
\text { mice }\end{array}$} & \multicolumn{4}{|c|}{ Antitumor activity over untreated animals } \\
\hline & & & & & $\begin{array}{l}\text { Median } \\
\text { survival } \\
\text { in days* }\end{array}$ & $\begin{array}{l}\text { Hazard } \\
\text { ratio }\end{array}$ & $\begin{array}{c}95 \% \\
\text { Confidence } \\
\text { limits }\end{array}$ & $\begin{array}{l}\text { Survival } \\
\text { curve } P^{\dagger}\end{array}$ \\
\hline 1 & 37 & +2 & IGROV-1 & 6 & $33 / 17(235)$ & 0.31 & $0.04-0.45$ & 0.0014 \\
\hline 2 & 37 & +4 & IGROV-1 & 8 & $15 / 10(150)$ & 0.37 & $0.03-0.62$ & 0.0105 \\
\hline \multirow[t]{2}{*}{3} & 37 & +2 & OVCAR3 & 8 & $34 / 14(239)$ & 0.23 & $0.01-0.16$ & $<0.0001$ \\
\hline & 37 & +4 & & 8 & $31 / 14$ (221) & 0.23 & $0.01-0.16$ & $<0.0001$ \\
\hline \multirow[t]{2}{*}{4} & 37 & +2 & OVCAR3 & 6 & $30 / 22$ (140) & 0.23 & $0.01-0.26$ & 0.0006 \\
\hline & 55 & +2 & & 4 & 35/22 (160) & 0.36 & $0.03-0.88$ & 0.0347 \\
\hline 5 & 74 & +2 and +9 & OVCAR3 & 6 & $\begin{array}{c}>90 / 19 \\
\text { (not reached) }\end{array}$ & 0.13 & $0.00-0.24$ & 0.0008 \\
\hline
\end{tabular}

*By 2-sided log-rank test over control mice.

${ }^{\dagger}$ Ratio of median survival time in treated over control mice $\times 100$.

Number of animals in controls ranged from 6 to 8 . Data in parentheses are percentages.

dimer format might stabilize binding as soon as the antigen-antibody complex is formed on the target tumor.

The systemic administration of the ${ }^{131}$ I-labeled human antibody fragment AFRA-DFM5.3 in animals bearing subcutaneous tumors, and comparison of its tumor uptake and therapeutic activity with those of the ${ }^{131}$ I-labeled nonbinding derivative AFRA-DFM6.1, allowed the definition of its therapeutic potential against FR-expressing tumors. Subsequent evaluation of ${ }^{131}$ I-AFRA-DFM5.3 in intraperitoneal tumor models of ovarian cancer mimicking a clinical situation demonstrated a strong efficacy against ovarian carcinoma.

In a mouse setting, an antibody fragment of human origin, even if suitable to limit immunogenic reactions in a future clinic situation, might result in suboptimal efficacy due to accelerated pharmacokinetics. In fact, the chemical-physical characteristics of antibodies are species-specific, and there is growing evidence that these parameters can contribute to their stability in circulation and alter the pharmacokinetic characteristics (24). Both AFRA-DFMs, in agreement with their human origin, exhibited a basic pI, and this parameter, together with the dimension of the AFRA-DFMs, could account for their short in vivo half-lives.

In animals not bearing tumors, similar pharmacokinetics were observed between AFRA-DFM5.3 and the nonbinder AFRA-DFM6.1, suggesting that the 2 AFRA-DFM human fragments are potentially able to discriminate between targeted and nontargeted effects of radioimmunotherapy. This aspect was examined using a well-defined subcutaneous tumor model system (21). In this in vivo system, despite a rapid elimination, only ${ }^{131}$ I-AFRA-DFM5.3- that is, the radiolabeled dimer specifically recognizing FR on tumor cells-localized to FR-positive tumor masses in an amount sufficient to significantly slow tumor growth. Even after a second treatment, the intravenous administration of ${ }^{131}$ I-AFRA-DFM5.3 was unable to completely eradicate subcutaneous tumor masses. In our previous report, when tumor uptake of ${ }^{131}$ I-AFRA-DFM5.3 was measured in tumors at levels 10-fold higher than those in the present study, we observed specific but less intense tumor accumulation (4). In agreement with the experiments of Goldenberg's group (25) demonstrating that tumor dimension is important for success of radioimmunotherapy and our preliminary observations (data not shown), we would expect a higher uptake in the experimental conditions tested (very small tumor dimensions), although this was not the case. We suggest that the rapid blood clearance more than poor tumor penetration limits the potential of systemically administered ${ }^{131}$ I-radiolabeled human fragment to control solid subcutaneous tumor masses in the mouse model.

Thus, having obtained proof of specificity of ${ }^{131}$ I targeting by AFRA-DFM5.3 in the subcutaneous model system, we decided to confirm the specificity by locoregional administration exploiting 2 different intraperitoneal human carcinoma models and to evaluate radioimmunotherapy efficacy.

After intraperitoneal administration in tumor-bearing animals, the pharmacokinetics of ${ }^{131}$ I-AFRA-DFM5.3 were longer than those of the same reagent after intravenous delivery and of ${ }^{131}$ I-AFRA-DFM6.1 intravenously or intraperitoneally injected, whereas biodistribution in normal tissues of both AFRA-DFMs was similar to that observed after systemic administration for ${ }^{131}$ I-AFRA-DFM5.3 (4). A slower release of the antibody fragment specifically bound to the intraperitoneal tumor or ascitic cells might explain the longer blood terminal half-life of intraperitoneally administered ${ }^{131}$ I-AFRA-DFM5.3. As expected, when both ascitic tumor cells and a solid mass were present, significantly higher uptake was observed on freefloating cells because of their easier accessibility; however, when only the solid mass was present, the \%ID/g was similar to the higher one. The difference in FR levels between OVCAR3 and IGROV-1 did not lead to a difference in 


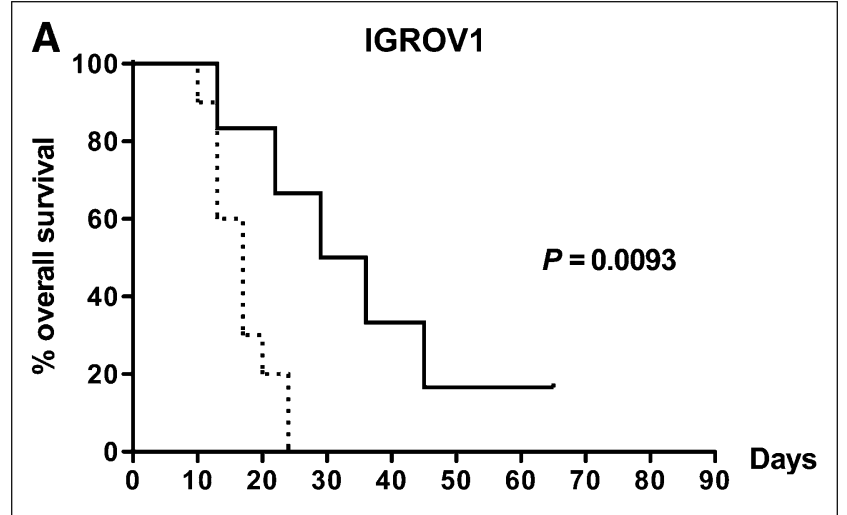

-..- Control
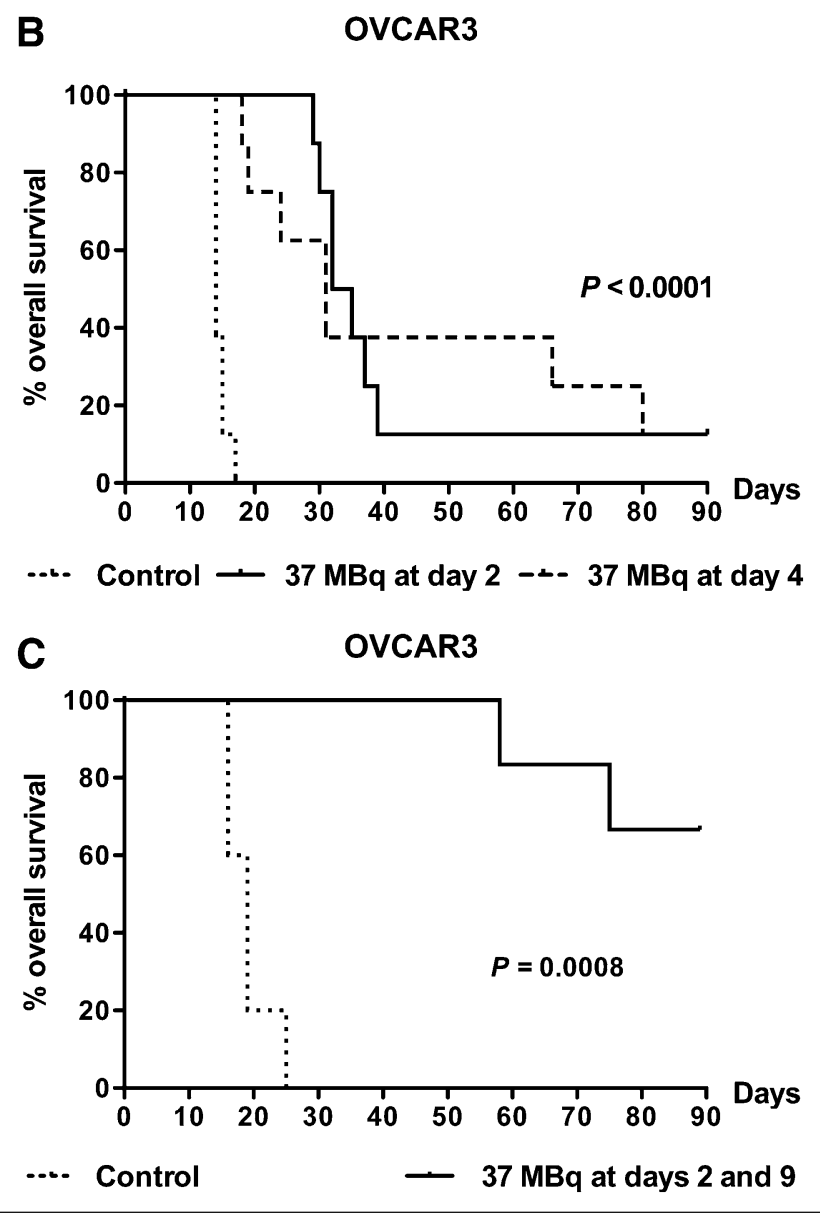

FIGURE 4. Efficacy of ${ }^{131} \mathrm{I}$-AFRA-DFM5.3 radioimmunotherapy administered by locoregional injection in intraperitoneal IGROV-1- or OVCAR3-bearing animals. (A) Single treatment with $37 \mathrm{MBq}$ administered on day 2 in intraperitoneal IGROV-1-bearing animals. (B) Single treatment with $37 \mathrm{MBq}$ administered on day 2 or day 4 in intraperitoneal OVCAR3-bearing animals. (C) Double treatment 1 wk apart, with a total of $74 \mathrm{MBq}$ in intraperitoneal OVCAR3-bearing animals; Table 2 provides details and statistical analysis. Survival curves are shown; $P$ values were calculated with 2 -sided log-rank test over control mice.

tumor uptake (data not shown). Furthermore, in both intraperitoneal models, a significant difference in tumor uptake was observed between the specific targeting of ${ }^{131}$ I-AFRA-
DFM5.3 and the nonspecific accumulation of ${ }^{131}$ I-AFRADFM6.1. However, in the absence of ascitic fluid, possibly because of a slow fluid recirculation in the peritoneal cavity, the nonspecific uptake was quite relevant, suggesting that, in these experimental conditions, a nontargeted effect could also be recorded. For this reason, radioimmunotherapy experiments were performed only with the specific binding dimer-that is, ${ }^{131}$ I-AFRA-DFM5.3.

It should be stressed that the efficacy seen in preclinical models must be extremely promising if the agent is to be considered clinically. In fact, the clinical effects are generally less impressive than preclinical data. Thus, it is important to perform accurate pharmacokinetics and biodistribution analyses. Our data after intraperitoneal administration highlight the relevance of early and facile access to tumor cells in determining the success of radioimmunotherapy with ${ }^{131}$ I-AFRA-DFM5.3 and, accordingly, the optimal antitumor activity, with more than $50 \%$ of animals cured of intraperitoneal tumors - that is, tumor-free at necropsy-was achieved after 2 locoregional administrations. On the basis of biodistribution data, it cannot be excluded that nontargeted effects may contribute to the therapeutic efficacy of targeted intraperitoneal treatment, confirming that nonspecific tumor irradiation (cross-fire effect) could by useful for tumor eradication.

The encouraging results obtained with double administration of intraperitoneal treatment suggest that additional schedules of delivery should be explored. In fact, dose fractionation has been proposed to improve the therapeutic effects of radioimmunotherapy $(26,27)$. Furthermore, because radioimmunotherapy is able to modify vascular permeability and tumor hypoxia, favoring the following pharmacologic therapy treatment (28-30), combined treatments could be envisioned.

Furthermore, the favorable biologic characteristics of FR make it an attractive target for ovarian cancer radioimmunotherapy. FR is overexpressed on $90 \%$ of ovarian carcinoma (31), whereas it is largely absent in normal tissues. FR expression, on normal epithelial cells such as in kidney proximal tubules, breast, and choroid plexus (32), is restricted to the apical (luminal) surface of polarized cells, on which it is not exposed to the bloodstream and not accessible to antibody (33). Moreover, expression of FR is associated with tumor progression in ovarian carcinoma (34); and, importantly, on the basis of published (35) and unpublished data, the expression of the FR is stable or even upmodulated during acquisition of drug resistance. In support of the mentioned preclinical and clinical data, the humanized anti-FR $\mathrm{mAb}$ farletuzumab is now in phase II and III clinical trials in combination with chemotherapy (36).

Overall, the present results reinforce previous preclinical in vitro evaluations, further supporting the good affinity and specificity of AFRA-DFM5.3 for the FR overexpressed in ovarian cancer and demonstrating the suitability of 2 locoregional administrations of radiolabeled antibody fragments in eradicating ovarian cancer cells and significantly prolonging survival of tumor-bearing mice. 


\section{CONCLUSION}

The human origin of AFRA-DFM5.3 and its efficacy when delivered locoregionally as an ${ }^{131}$ I reagent, together with evidence of the feasibility and acceptable toxicity profile of ovarian cancer treatment with anti-FR mAbs, could provide the basis for rational design of new therapeutic modalities. ${ }^{131}$ I-AFRA-DFM5.3, because it acts with a mechanism that is different from current treatments, may be a good therapeutic alternative for patients with ovarian cancer, to eradicate minimal residual disease by locoregional adjuvant administration.

\section{DISCLOSURE STATEMENT}

The costs of publication of this article were defrayed in part by the payment of page charges. Therefore, and solely to indicate this fact, this article is hereby marked "advertisement" in accordance with 18 USC section 1734.

\section{ACKNOWLEDGMENTS}

We thank Paola Alberti and Renata Ferri for technical assistance and Michele De Paola for help with artwork. This work was supported in part by the European project Magnifyco (contract NMP4-SL-2009-228622). No other potential conflict of interest relevant to this article was reported.

\section{REFERENCES}

1. Jemal A, Siegel R, Xu J, Ward E. Cancer statistics, 2010. CA Cancer J Clin. 2010;60:277-300.

2. Ledermann JA, Raja FA. Targeted trials in ovarian cancer. Gynecol Oncol. 2010;119:151-156

3. Weiner LM, Surana R, Wang S. Monoclonal antibodies: versatile platforms for cancer immunotherapy. Nat Rev Immunol. 2010;10:317-327.

4. Figini M, Martin F, Ferri R, et al. Conversion of murine antibodies to human antibodies and their optimization for ovarian cancer therapy targeted to the folate receptor. Cancer Immunol Immunother. 2009;58:531-546.

5. Koppe MJ, Postema EJ, Aarts F, Oyen WJ, Bleichrodt RP, Boerman OC. Antibodyguided radiation therapy of cancer. Cancer Metastasis Rev. 2005;24:539-567.

6. Knogler K, Grunberg J, Zimmermann K, et al. Copper-67 radioimmunotherapy and growth inhibition by anti-L1-cell adhesion molecule monoclonal antibodies in a therapy model of ovarian cancer metastasis. Clin Cancer Res. 2007;13:603-611.

7. Goldsmith SJ. Radioimmunotherapy of lymphoma: Bexxar and Zevalin. Semin Nucl Med. 2010;40:122-135.

8. Carter PJ. Potent antibody therapeutics by design. Nat Rev Immunol. 2006;6:343-357.

9. Lengyel E. Ovarian cancer development and metastasis. Am J Pathol. 2010;177:1053-1064.

10. Singhal $P$, Lele $S$. Intraperitoneal chemotherapy for ovarian cancer: where are we now? J Natl Compr Canc Netw. 2006;4:941-946.

11. Crippa F, Bolis G, Seregni E, et al. Single dose intraperitoneal radioimmunotherapy with the murine monoclonal antibody ${ }^{131} \mathrm{I}-\mathrm{MOv} 18$ : clinical results in patients with minimal residual disease of ovarian cancer. Eur $J$ Cancer. 1995;31A:686-690.

12. Canevari S, Stoter G, Arienti F, et al. Regression of advanced ovarian carcinoma by intraperitoneal treatment with autologous T-lymphocytes retargeted by a bispecific monoclonal antibody. J Natl Cancer Inst. 1995;87:1463-1469.

13. Canevari S, Mezzanzanica D, Mazzoni A, et al. Bispecific antibody targeted $\mathrm{T}$ cell therapy of ovarian cancer: clinical results and future directions. J Hematother. 1995;4:423-427.
14. Coney LR, Mezzanzanica D, Sanborn D, Casalini P, Colnaghi MI, Zurawski VR Jr. Chimeric murine-human antibodies directed against folate binding receptor are efficient mediators of ovarian carcinoma cell killing. Cancer Res. 1994;54:2448-2455.

15. Molthoff CF, Buist MR, Kenemans P, Pinedo HM, Boven E. Experimental and clinical analysis of the characteristics of a chimeric monoclonal antibody, MOv18, reactive with an ovarian cancer-associated antigen. J Nucl Med. 1992;33:2000-2005.

16. Buist MR, Kenemans P, Den Hollander W, et al. Kinetics and tissue distribution of the radiolabeled chimeric monoclonal antibody MOv18 IgG and $\mathrm{F}\left(\mathrm{ab}^{\prime}\right)_{2}$ fragments in ovarian carcinoma patients. Cancer Res. 1993;53:5413-5418.

17. Buijs WCAM, Massuger LFAG, Claessens RAMJ, Kenemans P, Corstens FHM. Dosimetric evaluation of immunoscintigraphy using indium-111-labeled monoclonal antibody fragments in patients with ovarian cancer. $J$ Nucl Med. 1992;33:1113-1120.

18. van Zanten-Przybysz I, Molthoff CF, Roos JC, et al. Influence of the route of administration on targeting of ovarian cancer with the chimeric monoclonal antibody MOv18: i.v. vs. i.p. Int J Cancer. 2001;92:106-114.

19. Figini M, Obici L, Mezzanzanica D, et al. Panning phage antibody libraries on cells: isolation of human Fab fragments against ovarian carcinoma using guided selection. Cancer Res. 1998;58:991-996.

20. Zacchetti A, Coliva A, Luison E, et al. ${ }^{177} \mathrm{Lu}$ - labeled MOv18 as compared to ${ }^{131} \mathrm{I}$ - or ${ }^{90}$ Y-labeled MOv18 has the better therapeutic effect in eradication of alpha folate receptor-expressing tumor xenografts. Nucl Med Biol. 2009;36:759770 .

21. Coliva A, Zacchetti A, Luison E, et al. ${ }^{90} \mathrm{Y}$ Labeling of monoclonal antibody MOv18 and preclinical validation for radioimmunotherapy of human ovarian carcinomas. Cancer Immunol Immunother. 2005;54:1200-1213.

22. Workman P, Aboagye EO, Balkwill F, et al. Guidelines for the welfare and use of animals in cancer research. Br J Cancer. 2010;102:1555-1577.

23. Tijink BM, Neri D, Leemans CR, et al. Radioimmunotherapy of head and neck cancer xenografts using ${ }^{131} \mathrm{I}$-labeled antibody L19-SIP for selective targeting of tumor vasculature. J Nucl Med. 2006;47:1127-1135.

24. Kobayashi H, Le N, Kim IS, et al. The pharmacokinetic characteristics of glycolated humanized anti-Tac Fabs are determined by their isoelectric points. Cancer Res. 1999;59:422-430.

25. Sharkey RM, Goldenberg DM. Perspectives on cancer therapy with radiolabeled monoclonal antibodies. J Nucl Med. 2005;46(suppl 1):115S-127S.

26. Elgqvist J, Andersson H, Back $\mathrm{T}$, et al. Fractionated radioimmunotherapy of intraperitoneally growing ovarian cancer in nude mice with ${ }^{211}$ At-MX35 F(ab')2: therapeutic efficacy and myelotoxicity. Nucl Med Biol. 2006;33:10651072 .

27. Dearling JL, Pedley RB. Technological advances in radioimmunotherapy. Clin Oncol (R Coll Radiol). 2007;19:457-469.

28. Blumenthal RD, Kashi R, Sharkey RM, Goldenberg DM. Quantitative and qualitative effects of experimental radioimmunotherapy on tumor vascular permeability. Int J Cancer. 1995;61:557-566.

29. Blumenthal RD, Sharkey RM, Kashi R, Sides K, Stein R, Goldenberg DM. Changes in tumor vascular permeability in response to experimental radioimmunotherapy: a comparative study of 11 xenografts. Tumour Biol. 1997;18: 367-377.

30. Blumenthal RD, Taylor A, Osorio L, et al. Optimizing the use of combined radioimmunotherapy and hypoxic cytotoxin therapy as a function of tumor hypoxia. Int J Cancer. 2001;94:564-571.

31. Miotti S, Canevari S, Ménard S, et al. Characterization of human ovarian carcinoma-associated antigens defined by novel monoclonal antibodies with tumorrestricted specificity. Int J Cancer. 1987;39:297-303.

32. Stein R, Goldenberg DM, Mattes MJ. Normal tissue reactivity of four anti-tumor monoclonal antibodies of clinical interest. Int J Cancer. 1991;47:163-169.

33. Weitman SD, Lark RH, Coney LR, et al. Distribution of the folate receptor GP38 in normal and malignant cell lines and tissues. Cancer Res. 1992;52: 3396-3401.

34. Toffoli G, Cernigoi C, Russo A, Gallo A, Bagnoli M, Boiocchi M. Overexpression of folate binding protein in ovarian cancers. Int J Cancer. 1997; 74:193-198.

35. Ottone F, Miotti S, Bottini C, et al. Relationship between folate binding protein expression and cisplatin sensitivity in ovarian carcinoma cell lines. Br J Cancer. 1997;76:77-82.

36. Spannuth WA, Sood AK, Coleman RL. Farletuzumab in epithelial ovarian carcinoma. Expert Opin Biol Ther. 2010;10:431-437. 